\title{
Surgical treatment of iatrogenic pseudoaneurysms
}

\author{
Hakan Kara (D) \\ Department of Cardiovascular Surgery, Giresun Ada Hospital, Giresun, Turkey
}

\begin{abstract}
Objectives: This study aims to present the results of surgical treatment of femoral and brachial artery pseudoaneurysms after cardiac catheterization.

Patients and methods: This retrospective study included a total of 28 patients (10 males, 18 females; mean age $76.5 \pm 11.5$ years; range, 52 to 90 years) who were diagnosed with pseudoaneurysms between January 2012 and December 2018. Diagnosis of pseudoaneurysm was made based on physical examination and arterial Doppler ultrasonography findings in all patients. The patients with a pseudoaneurysm of $\geq 2 \mathrm{~cm}$ in diameter underwent surgery.

Results: Two patients developed a brachial pseudoaneurysm, while 26 patients developed a femoral pseudoaneurysm. The location of the femoral pseudoaneurysm was the superficial femoral artery in 19 (73.07\%), common femoral artery in five (19.23\%), and deep femoral artery in two $(7.69 \%)$ patients. No complications were observed during follow-up.

Conclusion: Various treatment modalities can be used for iatrogenic pseudoaneurysms. Surgical treatment of iatrogenic pseudoaneurysms is a simple and successful method.
\end{abstract}

Keywords: Brachial artery, cardiac catheterization, femoral artery, open surgery, pseudoaneurysm.

A pseudoaneurysm is defined as a defect in the arterial wall which allows communication of the arterial blood with the adjacent soft tissues and compressed thrombus, thereby, forming a sac. ${ }^{[1]}$ The difference between pseudoaneurysm and true aneurysm is that the former does not include all of the arterial wall layers. The incidence of femoral pseudoaneurysms following interventional procedures varies between 0.2 and $6 \% .{ }^{[2]}$ They are an uncommon clinical entity, often arising after a trauma or surgical procedure.

Peripheral arterial pseudoaneurysms due to traumatic causes are not common, while development of pseudoaneurysms is more common owing to the increasing number of arterial punctures performed for diagnosis and treatment purposes in parallel with the advances in invasive cardiological interventional methods. This results in prolonged hospital stays, increased costs, and significant morbidity. Therefore, treating iatrogenic pseudoaneurysms is critical due to the risks of complications. Factors such as prolonged procedures, use of large-diameter interventional cannula, obesity, thrombolytic and antiplatelet drug use, hypertension, inability to have common femoral artery punctures, multiple arterial punctures, and short-term non-effective compression increase the rate of iatrogenic pseudoaneurysms. ${ }^{[3]}$

In this study, we aimed to present the results of surgical treatment of femoral and brachial artery pseudoaneurysms after cardiac catheterization.

\section{PATIENTS AND METHODS}

Between January 2012 and December 2018, a total of 10,643 patients underwent cardiac catheterization for the diagnosis and treatment at cardiovascular surgery clinic of Giresun Ada Hospital were screened. Data of a total of 28 patients (10 males, 18 females; mean age $76.5 \pm 11.5$ years; range, 52 to 90 years) who developed 
femoral artery and brachial artery pseudoaneurysms after cardiac angiography were retrospectively analyzed. A written informed consent was obtained from each patient. The study protocol was approved by the Ethics Committee of Karadeniz Technical University, Faculty of Medicine (15.02.2019/25). The study was conducted in accordance with the principles of the Declaration of Helsinki.

Diagnosis of pseudoaneurysm was established based on physical examination and Doppler ultrasonography (US) findings. All patients suffered from pain and hematoma at the cardiac catheterization site. Doppler US was adequate in establishing the diagnosis in all patients (Figure 1). Pseudoaneurysms of $<2 \mathrm{~cm}$ in diameter were treated non-surgically and were followed by regular ultrasonographic examination at the outpatient clinic. The patients with a pseudoaneurysm with a diameter of $\geq 2 \mathrm{~cm}$ underwent surgery. The patients with deep and superficial femoral artery aneurysms due to risk of rupture and pseudoaneurysms greater than $5 \mathrm{~cm}$ were operated as early as possible. Those who developed pseudoaneurysms owing to other reasons were excluded from the study.

Angiography was performed with a $6 \mathrm{~F}$ sheath for the femoral artery and with a $5 \mathrm{~F}$ sheath and standard Judkins catheters (Boston Scientific, Marlborough, MA, USA) for the brachial artery. Prior to the procedure, antiplatelet treatment was started with 300 to $450 \mathrm{mg}$ clopidogrel for patients who underwent cardiac angiography due to acute coronary syndrome. During angiography, 5,000 to 7,500 U of unfractionated heparin was administered to patients prior to stent placement. Following angiography, a pneumatic compression device was applied to the

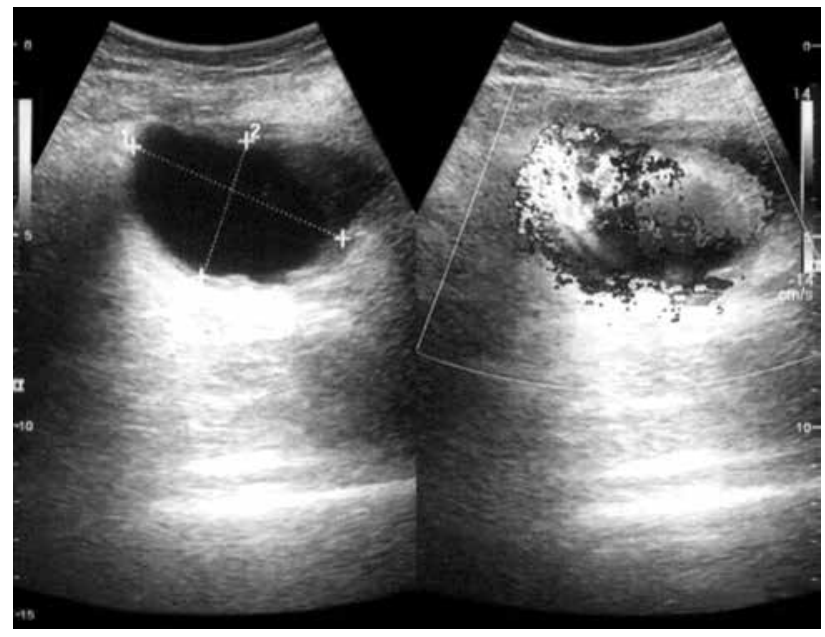

Figure 1. Doppler ultrasonography showing a femoral pseudoaneurysm. patients for four $h$, and they were recommended bed rest for a total of six h, including two h after deflating the balloon of the device. The patients who received stents were kept under observation for at least $24 \mathrm{~h}$, and the sheath was removed six $\mathrm{h}$ after the procedure. In patients in whom cardiac angiography decision was medical, the sheath was removed immediately after angiography was performed, and the patients were discharged six $h$ later with necessary recommendations. One patient underwent emergency surgery due to rupture. Patients with brachial pseudoaneurysms were operated under local anesthesia (Figure 2), whereas those with femoral aneurysms were operated under spinal anesthesia. Heparinization was performed with $100 \mathrm{U} / \mathrm{kg}$ standard heparin, which was administered intravenously, by maintaining the activated clotting time of over $200 \mathrm{sec}$. In eligible cases, the main femoral, superficial femoral, deep femoral, and brachial arteries were suspended with tapes, whereas exploration was achieved by applying compression proximally to the pseudoaneurysm in ineligible patients. The patients with massive hemorrhage and complicated pseudoaneurysms were not prepared for proximal and distal control of the femoral artery. It was directly inserted into the pseudoaneurysm sac and bleeding was controlled by finger. A $5 \mathrm{~F}$ Fogarty arterial embolectomy catheter was inserted from the injured site and the artery bleeding was controlled by the balloon occlusion method. The bleeding site in the artery was primarily repaired using $5 / 0$ monofilament polypropylene suture material. The pseudoaneurysm sac was resected after emptying the hematoma. After achieving control over the bleeding, the surgical site was washed with ample amounts of saline solution. A Hemovac drain was placed, and tissues were closed

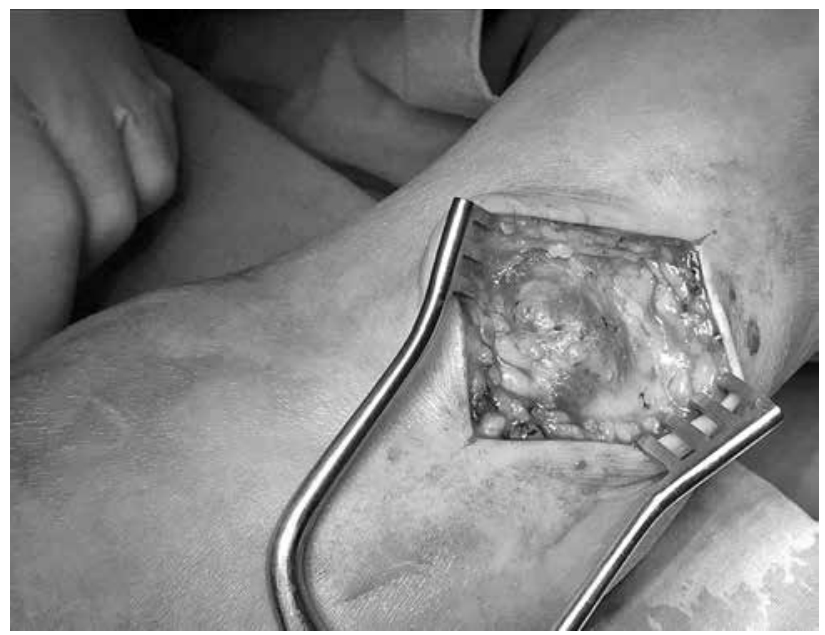

Figure 2. An intraoperative view of a brachial pseudoaneurysm. 
in layers. In patients who underwent coronary artery surgery in addition to pseudoaneurysm operation, surgery for pseudoaneurysm was performed initially, followed by coronary artery bypass grafting (CABG). The patients were followed at the end of the first month with Doppler US for persistent pseudoaneurysms or any other complications.

\section{Statistical analysis}

Statistical analysis was performed using the IBM SPSS version 23.0 software (IBM Corp., Armonk, NY, USA). Continuous variables were expressed in mean \pm standard deviation (SD) and median (min-max), whereas categorical variables were expressed in number and frequency.

\section{RESULTS}

Of all patients, two (7.14\%) developed a brachial pseudoaneurysm, while 26 (92.86\%) developed a femoral pseudoaneurysm. Overall, 26 patients (92.86\%) had hypertension, 10 (35.71\%) had diabetes mellitus, 11 (39.28\%) had dyslipidemia, and two (7.14\%) had peripheral artery disease. Baseline demographic and clinical characteristics of the patients are presented in Table 1.

The reason of admission for all patients was swelling and pain in the groin and arm regions. During physical examination, a painful and pulsatile mass was detected in all patients. The mean pseudoaneurysm size was $5.9 \pm 1.9 \mathrm{~cm}$. The location of femoral pseudoaneurysm was the superficial femoral artery in 19 (73.07\%), common femoral artery in five (19.23\%), and deep femoral artery in two patients (7.69\%) (Table 2). Two pseudoaneurysms in the arm region were localized

Table 1. Demographic and clinical characteristics of patients

\begin{tabular}{lcc}
\hline & $\mathrm{n}$ & $\%$ \\
\hline Gender & & \\
$\quad$ Male & 10 & 35.71 \\
$\quad$ Female & 18 & 64.29 \\
Hypertension & 26 & 92.85 \\
Diabetes mellitus & 10 & 35.71 \\
Dyslipidemia & 11 & 39.28 \\
Peripheral artery disease & 2 & 7.14 \\
\hline
\end{tabular}

Table 2. Location of femoral pseudoaneurysm

\begin{tabular}{lcc}
\hline & $\mathrm{n}$ & $\%$ \\
Superficial femoral artery & 19 & 73.07 \\
Common femoral artery & 5 & 19.23 \\
Deep femoral artery & 2 & 7.69 \\
\hline
\end{tabular}

in the brachial artery. Based on cardiac angiography results, the decision to perform CABG was made in three patients and to place a coronary artery stent in five patients. The coronary artery was normal in 20 patients. In three patients for whom surgery was decided, CABG and pseudoaneurysm surgery were performed simultaneously. The mean time to discharge was $2.1 \pm 0.6$ (range, 2 to 4 ) days, except for those who underwent CABG. One patient underwent surgery under emergency conditions due to a clinical presentation of shock associated with ruptured pseudoaneurysm. Infection, distal embolism, ischemia, and mortality were not observed in any of the patients. No persistent pseudoaneurysm was observed during follow-up as confirmed by Doppler US at one month after surgery.

\section{DISCUSSION}

A pseudoaneurysm is a pulsatile mass caused by the deterioration of the integrity of the arterial wall and is associated with the vessel wall. ${ }^{[4]}$ The incidence of femoral pseudoaneurysms following interventional procedures varies between $0.2 \%$ and $6 \%{ }^{[2]}$ In recent years, there has been an increase in the incidence of pseudoaneurysms, particularly femoral pseudoaneurysms, as diagnostic methods have become widespread. In the study conducted by Erol et al. ${ }^{[4]}$ in 8,649 patients who underwent cardiac catheterization, coronary and/or peripheral angiography, and percutaneous coronary interventions via the femoral artery, the prevalence of pseudoaneurysm was found to be $65(0.76 \%)$. In the study conducted by Huseyin et al., ${ }^{[5]}$ among 12,261 patients who underwent percutaneous intervention for cardiac catheterization, 55 patients $(0.44 \%)$ developed femoral artery pseudoaneurysm and $42(0.34 \%)$ of them received surgical treatment.

Inadvertent catheterization of the superficial femoral artery or profunda femoris artery, interventional rather than diagnostic procedures, inadequate compression following the removal of the arterialsheath, obesity, hypertension, calcified arteries, aberrant anatomy with high branching common femoral artery, and the need for post-procedural anticoagulation can be considered the main causes of pseudoaneurysm development. ${ }^{[2]}$ Common femoral artery puncture is optimal for arterial catheterization due to the fact that the femoral sheath is robust all around at this level and the fact that the presence of the femur head behind it increases the effectiveness of compression therapy. Moreover, the walls of the 
superficial and deep femoral arteries are thinner than that of the common femoral artery. Thus, these arterial walls are easily damaged during interventional procedures, and pseudoaneurysms may occur easily. ${ }^{[3,5]}$ In our study, we found that iatrogenic femoral artery pseudoaneurysms mostly resulted from superficial femoral arteries in 19 patients (73.07\%). External iliac artery puncture should be avoided due to serious risk of hemorrhage in the retroperitoneal region. ${ }^{[3]}$ The rate of occurrence of pseudoaneurysm is less in brachial artery punctures. However, as they have the potential to cause serious complications, they require rapid diagnosis and treatment.

The clinical picture of pseudoaneurysms varies depending on their localization, size, and growth rate. Patients with femoral pseudoaneurysms typically present with pain and edema of the affected groin along with a palpable mass, which may be pulsatile with a thrill or bruit. However, in obese patients or in cases wherein pseudoaneurysm involves the profunda femoris artery, clinical diagnosis can be difficult. Small pseudoaneurysms may resolve spontaneously without any intervention. Conversely, persistent pseudoaneurysms may enlarge and lead to complications related to compression of the adjacent femoral vein, nerve, and overlying skin. This may cause leg edema, deep vein thrombosis, compressive neuropathy, skin necrosis, and rupture. ${ }^{[6]}$ Pseudoaneurysms of $>5 \mathrm{~cm}$ in diameter are associated with a high risk of rupture. ${ }^{[7]}$ In the current study, the most common complaints were painful and a pulsatile mass. In our study, one patient (3.57\%) underwent surgery under emergency conditions due to rupture. Doppler US is the modality of choice for diagnosing femoral pseudoaneurysms and has a calculated sensitivity of $94 \%$ and a specificity of $97 \%{ }^{[8]}$ The hallmark of diagnosis is the demonstration of a neck communicating between the sac and affected artery, with a to-and-fro waveform. Computed tomography angiography is also a valuable diagnostic tool. ${ }^{[6]}$ In our study, all patients were diagnosed using Doppler US. Although treatment is required to prevent complications in large symptomatic pseudoaneurysms, a close follow-up is needed for patients with pseudoaneurysms of $<2 \mathrm{~cm} .{ }^{[9]}$ Stone et al. ${ }^{[10]}$ conservatively followed 167 patients with pseudoaneurysms of $<3 \mathrm{~cm}$ and reported the need for additional intervention in $16 \%$ of these patients. Recently, methods other than surgery, such as ultrasound-guided compression, ultrasound-guided thrombin injection, biodegradable collagen injection, coil embolization, and placement of covered stents/ endoluminal prostheses have become popular for the treatment of pseudoaneurysms which develop after catheterization. ${ }^{[2,6,11]}$ The most commonly used nonsurgical method is ultrasound-guided compression or thrombin injection. These methods may be either advantageous or disadvantageous. However, they have disadvantages such as prolonged procedural time, significant pain during compression, a higher incidence of failure in large and complex pseudoaneurysms, and a higher recurrence rate in patients receiving anticoagulant therapy. ${ }^{[8,12]}$ Successful rate of thrombin injection has been reported as 86 to $92 \% \cdot{ }^{[13]}$ Additionally, thrombin injections may cause anaphylactic shock, pruritus, femoral artery thrombosis or embolism, femoral vein thrombosis, or infection within the aneurysm sac. ${ }^{[14]}$ Rarely, prion injections may cause chronic degenerative brain disease, as thrombin is of bovine origin. ${ }^{[15]}$ Mishra et al. ${ }^{[16]}$ reported that thrombin was injected in incremental small doses as mentioned above to avoid an accidental injection into the femoral artery. Among these different treatment options, the decision of the treatment to be opted for should be made by considering the size and localization of the pseudoaneurysm sac, surgical experience, and cost. In a study conducted by Akay et al. ${ }^{[17]}$ in a series of 97 patients with pseudoaneurysm, ultrasoundguided compression or glue injection was performed in 59 patients, whereas surgical intervention was performed in 38 patients. In eight patients (13.5\%) who did not undergo surgery earlier, further surgical intervention was required due to a large hematoma, femoral artery occlusion caused by glue, and persistence of pseudoaneurysm. In another study in 66 patients who developed an iatrogenic femoral pseudoaneurysm and who were scheduled for CABG, Stone et al. ${ }^{[18]}$ found the Doppler-guided thrombin injection and surgical repair treatment options to be $100 \%$ effective for pseudoaneurysms. Griviau et al. ${ }^{[19]}$ performed embolization with ultrasound-guided balloon-assisted cyanoacrylate glue in 23 patients with a femoral pseudoaneurysm and obtained successful results in 20 patients (86.9\%); however, pseudoaneurysm remained persistent in three patients (13.1\%). The most common complications in the surgical treatment of femoral artery pseudoaneurysms are bleeding and incision site infections. Piffaretti et al. ${ }^{[20]}$ reported a rate of $13 \%$ for hemorrhage and a rate of $6.5 \%$ for incision site infection as complications of the iatrogenic femoral artery pseudoaneurysm surgery. In our study, incision site infections did not develop in any patient. We believe that washing the surgical drain site and surgical area causing negative pressure with ample amounts of physiological saline solution decreases the 
occurrence of postoperative infections. In addition, no blood transfusion was required for any patient during the postoperative period, except for one patient for whom emergency surgery was performed due to pseudoaneurysm rupture. In our study, none of the patients developed distal embolism, venous thrombosis, or neurological deficits. Borioni et al. ${ }^{[21]}$ reported a mortality rate of $4.4 \%$ with surgical treatment of iatrogenic femoral artery pseudoaneurysms. Mortality was not reported for any patient included in our study. The reason for this is that patients underwent surgery in the shortest time possible, and meticulous and careful surgery was performed. Surgical closure of the femoral pseudoaneurysm is considered the gold standard for treatment of pseudoaneurysms. ${ }^{[15]}$ A simple suture of the defect after evacuating the hematoma may be enough; alternatively, a surgical repair with a patch can be performed. ${ }^{[3]}$ In this study, 26 patients with femoral and two with brachial artery pseudoaneurysm were surgically treated, and 100\% success was achieved by repairing the hole in the artery with a primary suture.

The limitations of present study were relatively small sample size and retrospective nature of the study.

In conclusion, pseudoaneurysms are serious complications which are associated with important morbidity and mortality such as local pressure, rupture, bleeding, infection and thrombosis. Pseudoaneurysms can be easily and successfully treated through a simple surgical technique under local or spinal anesthesia.

\section{Declaration of conflicting interests}

The author declared no conflicts of interest with respect to the authorship and/or publication of this article.

\section{Funding}

The author received no financial support for the research and/or authorship of this article.

\section{REFERENCES}

1. Saad NE, Saad WE, Davies MG, Waldman DL, Fultz PJ, Rubens DJ. Pseudoaneurysms and the role of minimally invasive techniques in their management. Radiographics 2005;25:173-89.

2. Ahmad F, Turner SA, Torrie P, Gibson M. Ahmad F, Turner SA, Torrie P, Gibson M. Clin Radiol 2008;63:1310-6.

3. Demirbas O, Batyraliev T, Eksi Z, Pershukov I. Femoral pseudoaneurysm due to diagnostic or interventional angiographic procedures. Angiology 2005;56:553-6.

4. Erol F, Arslan Ş, Yüksel İÖ, Üreyen ÇM, Serdar S, İnci S, et al. Determinants of iatrogenic femoral pseudoaneurysm after cardiac catheterization or percutaneous coronary intervention via the femoral artery. Turk Kardiyol Dern Ars 2015;43:513-9.

5. Huseyin S, Yuksel V, Sivri N, Gur O, Gurkan S, Canbaz $\mathrm{S}$, et al. Surgical management of iatrogenic femoral artery pseudoaneurysms: A 10-year experience. Hippokratia 2013;17:332-6.

6. Petrou E, Malakos I, Kampanarou S, Doulas N, Voudris $\mathrm{V}$. Life-threatening rupture of a femoral pseudoaneurysm after cardiac catheterization. Open Cardiovasc Med J 2016;10:201-4.

7. Levi N, Schroeder TV. True and anastomotic femoral artery aneurysms: is the risk of rupture and thrombosis related to the size of the aneurysms? Eur J Vasc Endovasc Surg 1999;18:111-3.

8. Morgan R, Belli AM. Current treatment methods for postcatheterization pseudoaneurysms. J Vasc Interv Radiol 2003;14:697-710.

9. Kırali K, Güler M, Mansuroğlu D, Ömeroğlu SN. Pseudoaneurysms of extremity arteries and treatments. Turk Gogus Kalp Dama 2000;8:802-4.

10. Stone PA, Martinez M, Thompson SN, Masinter D, Campbell JE, Campbell Ii JR, et al. Ten-year experience of vascular surgeon management of iatrogenic pseudoaneurysms: do anticoagulant and/or antiplatelet medications matter? Ann Vasc Surg 2016;30:45-51.

11. Hanson JM, Atri M, Power N. Ultrasound-guided thrombin injection of iatrogenic groin pseudoaneurysm: Doppler features and technical tips. Br J Radiol 2008;81:154-63.

12. Stone PA, AbuRahma AF, Flaherty SK, Bates MC. Femoral pseudoaneurysms. Vasc Endovascular Surg 2006;40:109-17.

13. Yoo T, Starr JE, Go MR, Vaccaro PS, Satiani B, Haurani MJ. Ultrasound-guided thrombin injection is a safe and effective treatment for femoral artery pseudoaneurysm in the morbidly obese. Vasc Endovascular Surg 2017;51:368-72.

14. Lisowska A, Knapp M, Usowicz-Szaryńska M, Kozieradzka A, Musial WJ, Dobrzycki S. Iatrogenic femoral pseudoaneurysms - a simple solution of inconvenient problem? Adv Med Sci 2011;56:215-21.

15. Tisi PV, Callam MJ. Surgery versus non-surgical treatment for femoral pseudoaneurysms. Cochrane Database Syst Rev 2006;1:CD004981.

16. Mishra A, Rao A, Pimpalwar Y. Ultrasound guided percutaneous injection of thrombin: Effective technique for treatment of iatrogenic femoral pseudoaneurysms. J Clin Diagn Res 2017;11:TC04-TC06.

17. Akay HT, Korun O, Aslım E, Harman A, Özyer ŞE, Özkan M, et al. Clinical experience in postangiographic iatrogenic femoral pseudoaneurysm management. Turk J Vasc Surg 2002;21:120-3.

18. Stone PA, Thompson SN, Hanson B, Masinter D. Management of iatrogenic pseudoaneurysms in patients undergoing coronary artery bypass grafting. Vasc Endovasc Surg 2016;50:217-20.

19. Griviau L, Chevallier O, Marcelin C, Nakai M, Pescatori L, Galland C, et al. Percutaneous ultrasound-guided balloon-assisted embolization of iatrogenic femoral artery pseudoaneurysms with Glubran ${ }^{\circledR} 2$ cyanoacrylate glue: 
safety, efficacy and outcomes. Quant Imaging Med Surg 2018;8:796-803.

20. Piffaretti G, Mariscalco G, Tozzi M, Rivolta N, Castelli $\mathrm{P}$, Sala A. Predictive factors of complications after surgical repair of iatrogenic femoral pseudoaneurysms.
World J Surg 2011;35:911-6.

21. Borioni R, Garofalo M, De Paulis R, Albano P, Caprara E, Fratticci L, et al. Surgical treatment of femoral artery pseudoaneurysms after cardiac catheterization. Minerva Chir 2008;63:277-82. 